\title{
SOLID TO LIQUID TRANSITION OF INVERSE FERROFLUIDS UNDER SHEAR
}

\author{
R. Saldivar-Guerrero ${ }^{1,3}$, R. Richter ${ }^{1}$, I. Rehberg ${ }^{1}$, N. Aksel $^{2}$, \\ L. Heymann ${ }^{2}$, O.S. Rodriguez-Fernández ${ }^{3}$ \\ 1 Experimentalphysik V, Universität Bayreuth, D-95440 Bayreuth, Germany \\ ${ }^{2}$ Lehrstuhl für Technische Mechanik und Strömungsmechanik, Universität Bayreuth, \\ D-95440 Bayreuth, Germany \\ ${ }^{3}$ Centro de Investigación en Química aplicada, 25100 Saltillo, Coahuila México
}

By dispersing microsized monodisperse polystyrene particles in a ferrofluid, an ideal magneto-rheological model fluid is created. Due to the dipolar interactions of the particles, the hybrid fluid shows solid-like behavior under the shear, i.e., one has to overcome an apparent yield stress before flow sets in. We compare this quantity with theoretical predictions for different magnetic fields and volume fractions of nonmagnetic particles.

Introduction. Common magnetorheological fluids (MRFs) are suspensions of micron-sized, magnetizable particles like carbonyl iron, dispersed in a nonmagnetic carrier liquid. They are capable to change their rheological properties under the influence of a magnetic field. Their flow behavior is typically described as a classical Bingham fluid, where the yield stress is a function of the applied field strength [1].

It is known that plain ferrofluids can also be transformed in MRFs by the suspension of non-magnetic particles, like micron-size polystyrene or silica spheres, which are available with a very narrow size distribution. These non-magnetic particles create a hole, which appears to possess a magnetic moment $m=-4 \pi \beta a^{3} H$, corresponding to the displaced fluid [2], where $a$ is the radius of spherical particles, and $H$ is the magnetic field. Moreover $\beta=\left(\mu_{\mathrm{r}}-1\right) /\left(2 \mu_{\mathrm{r}}+1\right)$ characterizes the effective permeability of the suspension, where $\mu_{\mathrm{r}}$ represents the relative permeability of the fluid.

First studies of inverse ferrofluids were undertaken by Skjeltorp [2], who investigated the structures formed in a monolayer film of polystyrene spheres of $10 \mu \mathrm{m}$ in diameter. The structures are similar to those found in magnetorheological fluids consisting of ferromagnetic particles dispersed in a non-magnetic carrier. These structures are responsible for the magnetorheological effect, because the chain-like orientation of the dispersed nonmagnetic particles along the field lines restricts the motion of the carrier fluid in flow direction. In this way the fluid experiences a transition from a "solid-like" behavior, when the chains or aggregates are mainly intact, to a "liquid-like" behavior when they are broken. Such magnetorheological effect in fluids containing polystyrene particles with an average diameter of $0.8 \mu \mathrm{m}$ were studied by Bossis and Lemaire [3]. They modeled the yield stress, utilizing the calculus of Klingenberg and Zukoski, which was originally derived for dielectric spheres [4].

The objective of this work was to study the magnetorheological effect of inverse ferrofluids for various magnetic fields using different volume fractions of monodisperse nonmagnetic polystyrene particles in rotational measurements. From the flow curves we extract the apparent yield stress and compare the results with the model proposed by Bossis et al. [3]. 


\section{Experimental.}

1.1. Materials. The nonmagnetic particles used in our experiments were monodisperse polystyrene particles of $10.8 \mu \mathrm{m}$ in diameter and a density of 1.05 $\mathrm{g} \cdot(\mathrm{ml})^{-1}$, supplied by Microbeads AS. They were dried in a freeze dry system and used without further treatment. The particle size distribution was measured using static light scattering (SLS). The mean diameter $D$ and the normalized polydispersity $I=\left(d_{90}-d_{10}\right) / d_{50}$ are $10.86 \mu \mathrm{m}$ and 0.143 , respectively.

We used the ferrofluid APG 512A, Lot. No. F083094CX from Ferrotec Co. It contains magnetite particles dispersed in synthetic hydrocarbon with a viscosity of $116 \mathrm{mPa} \cdot \mathrm{s}$, at $20^{\circ} \mathrm{C}$. The magnetization curve obtained in a SQUID magnetometer showed a saturation magnetization of $M_{\mathrm{s}}=27.68 \mathrm{kA} / \mathrm{m}$.

1.2. Sample preparation. The inverse ferrofluids were prepared by taking the dried polystyrene particles (in different amounts) and dispersing them in the ferrofluid. The system was alternately stirred for 10 to 15 minutes and sonicated for the same time until a homogeneous mixture was obtained. The volume fractions of non-magnetic particles, namely, $\phi=0.10,0.175$ and 0.25 , were defined from mass measurements. The obtained samples were stable against sedimentation for several hours. This was corroborated by taking X-ray pictures of a filled container. In this way an inhomogeneous absorption unveiled the progress of sedimentation.

1.3. Rheological measurements. A Physica MCR500 rheometer (Anton Paar) with the commercial magnetorheological cell PP 20/MR was used to measure the magnetorheological properties of inverse ferrofluids. The field was oriented perpendicular to the plates of the rheometer, and thus, perpendicular to the direction of the shear. The distance between the plates was adjusted to $0.3 \mathrm{~mm}$ in order to optimize the sensitivity. Rotational measurements were carried out shearing the sample at a constant shear rate of $100 \mathrm{~s}^{-1}$ at zero field for $30 \mathrm{~s}$ to distribute the particles uniformly. Next, the desired magnetic field is applied and maintained for another $30 \mathrm{~s}$ to obtain a static structure. The flow curve is measured in a controlled shear stress modus (CSS). Each of the rheological measurements was performed with a freshly prepared sample at $20^{\circ} \mathrm{C}$.

2. Results. Fig. 1 presents photographs of a sample of the inverse ferrofluid placed in between two glass plates separated $25 \mu \mathrm{m}$ apart from each other. The pictures were taken by means of a long distance microscope, enabling us to observe the sample in between a pair of Helmholtz coils. Fig. 1a gives a picture, where no magnetic field was applied. Fig. $1 b$ proves that a network of chains of polystyrene spheres does exist at a field of $H_{0}=41.4 \mathrm{kA} / \mathrm{m}$.

Next, we investigate the flow curves of the inverse ferrofluid at an applied magnetic field of $H_{0}=144.1 \mathrm{kA} / \mathrm{m}$ for different volume fractions of polystyrene (PS) spheres, as presented in Fig. 2. Whereas the plain ferrofluid shows a Newtonian behavior in the full range of the shear rate investigated, the addition of nonmagnetic particles changes the flow behavior drastically. The inverse ferrofluids show an apparent yield stress, which is characteristic for the magnetorheological fluids.

The non-linear flow curves in Fig. 2 indicate a shear thinning due to the breaking of agglomerates formed in the field direction. The flow curves are well described by solid lines, which give a fit to the Casson model

$$
\tau^{1 / 2}=\tau_{y}^{1 / 2}+(k \dot{\gamma})^{1 / 2} .
$$

Here $\tau, \tau_{y}$ and $\dot{\gamma}$ denote the shear stress, yield stress and shear rate, respectively. As can be seen from Fig. 2, $\tau_{y}$ increases with the volume fraction $\phi$ of the dispersed particles. This is a consequence of the larger number of chains. Moreover, the yield stress should increase with the applied magnetic field. 


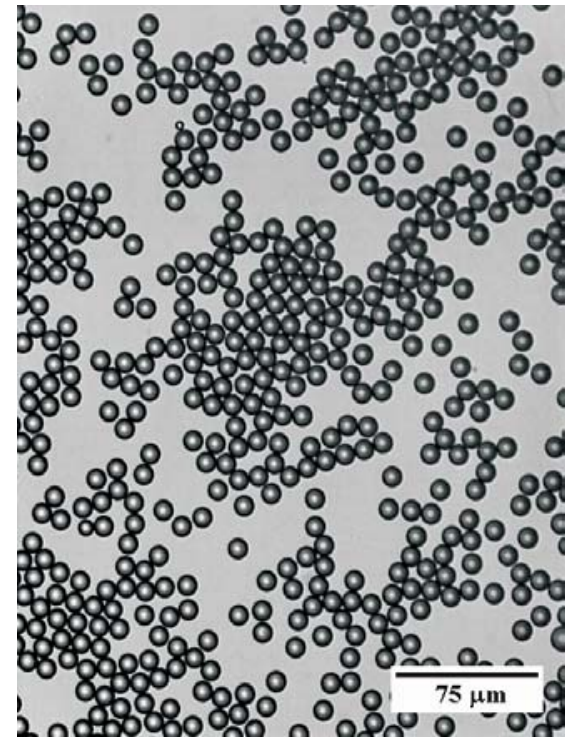

(a)

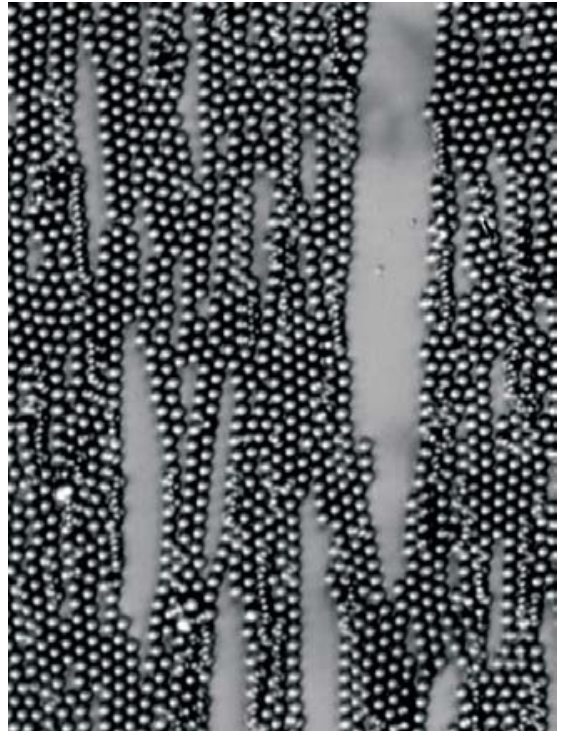

(b)

Fig. 1. Pictures of the structures formed in inverse ferrofluids with $\phi=0.175$ of particles for $(a)$ no magnetic field, and $(b) H_{0}=41.4 \mathrm{kA} / \mathrm{m}$, applied in the vertical direction.

We have investigated this effect for six different values of the applied magnetic field. Fig. $3 a$ shows the outcome. The symbols denote the yield stress obtained by fitting the flow curves with Eq. (1). It increases with the volume fraction and

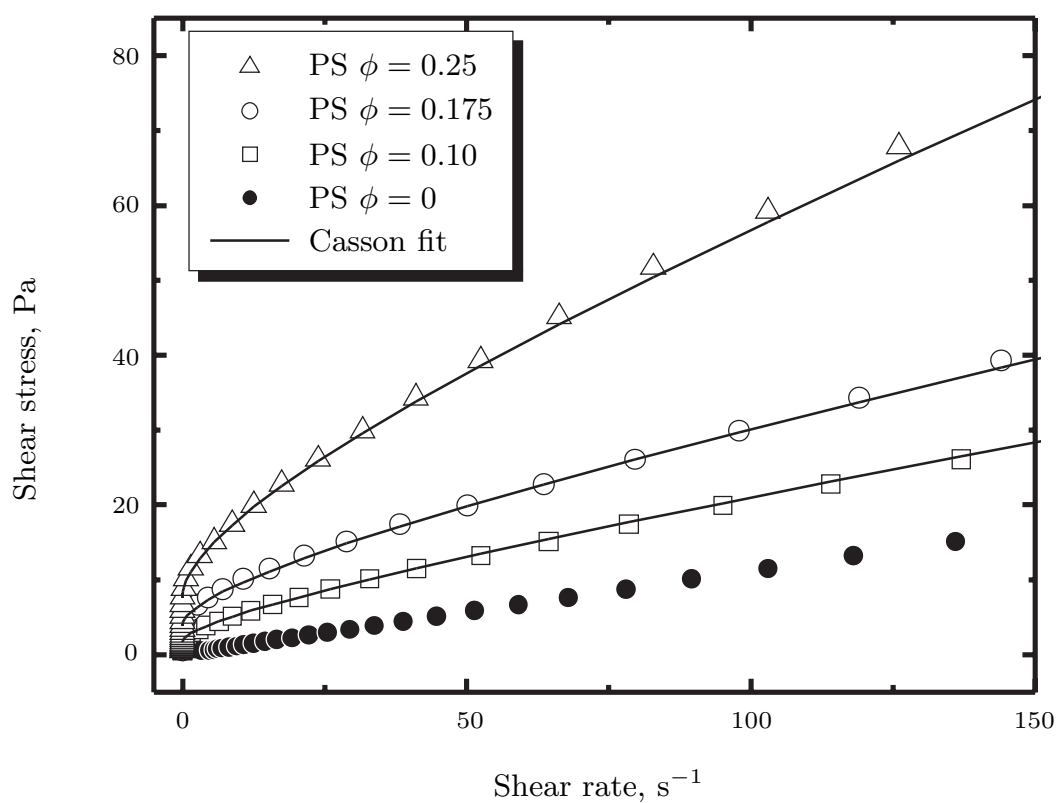

Fig. 2. The flow curves of inverse fluids with different volume fractions of non-magnetic particles at an externally applied magnetic field of $H_{0}=144.1 \mathrm{kA} / \mathrm{m}$. 

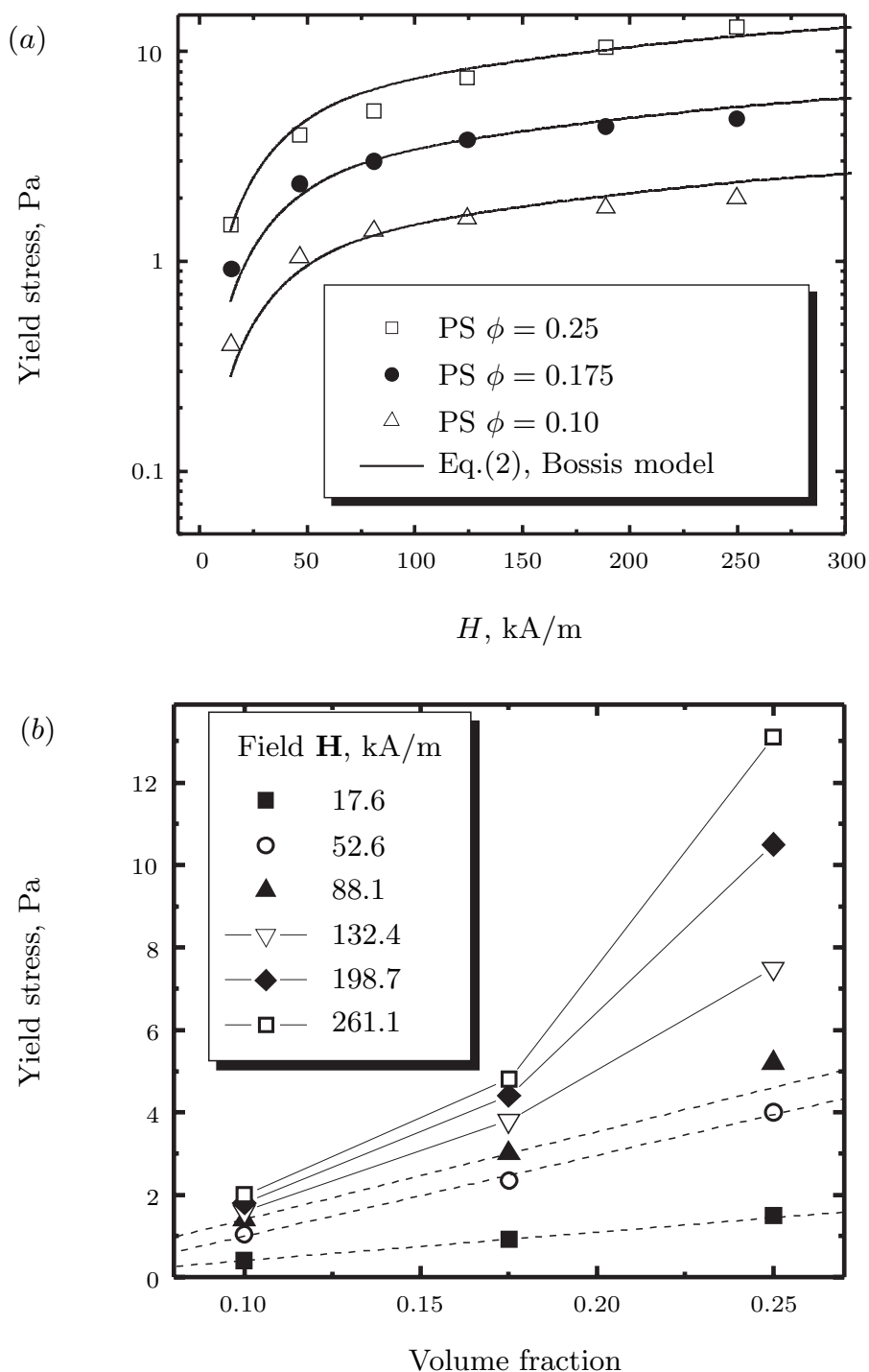

Fig. 3. Yield stress versus magnetic field as a function of the magnetic field $(a)$ and of the volume fraction of nonmagnetic particles $(b)$. The dashed lines give the linear extrapolation of the first two values.

the magnetic field. The solid lines display the fits by Bossis model according to

$$
\tau_{y}=\frac{3 \phi}{2 \pi a^{2}} F_{\mathrm{r}}^{\mathrm{m}}
$$

It calculates the maximum restoring force $F_{\mathrm{r}}^{\mathrm{m}}=3 \mu_{\mathrm{r}} a^{2} H^{2} \beta^{2} f(r, \theta)$, which tends to align two spheres on the axis of the field. This force depends linearly on the relative permeability $\mu_{\mathrm{r}}$, and quadratically on the radius $a$ of the spheres, the magnetic field $H$, and the effective permeability $\beta$. Here $H$ is the effective field in the sample between the two plates of the rheometer and depends on the externally applied magnetic field $H_{0}$, according to $H=H_{0} / \mu(H)$. Moreover the force is proportional to $f(r, \theta)=(a / r)^{4}\left[\left(f_{\|}+2 f_{\Gamma}\right) \sin \theta \cos ^{2} \theta-f_{\perp} \sin ^{3} \theta\right]$ given in [4]. This function depends on the separation $r$ of the two centers of the spheres and 
on the polar angle $\theta$ between the line of the centers and the axis of the magnetic field. In the dipolar limit for $\alpha=\mu_{\text {particle }} / \mu_{\text {fluid }}=1$ we have $f_{\|}=1, f_{\Gamma}=1$ and $f_{\perp}=1$ and a maximal angle $\theta_{\mathrm{m}}=21.3 \mathrm{deg}$ [4]. In our case $\alpha=0.46$, which is close to the dipolar limit. Thus, the Bossis model was applied for $r=2 a$ in the dipolar limit using $\theta$ as the fitting parameter. According to Fig. 3a, it describes well the experimental results. We obtained the values $\theta_{\mathrm{m}}=58.1,56.4$ and 52.1 for the volume fractions $\phi=0.1,0.175$ and 0.25 , respectively. Surprisingly, these values excess more than twice the theoretical value of $21.3 \mathrm{deg}$.

Independently from the dipolar approximation, $\tau_{y}$ should increase linearly with $\phi$, according to Eq. (2). Fig. $3 b$ is devoted to this question. For small magnetic fields this is indeed the case (dashed lines), whereas for moderate values $H>80 \mathrm{kA} / \mathrm{m}$ a deviation from the linear behavior can be observed.

3. Discussion and conclusion. We have investigated the magneorheological effect of inverse ferrofluids for different magnetic fields and volume fractions of nonmagnetic particles. The measurements can well be fitted by the model of Bossis et al. in the dipolar approximation. However, the obtained values for the angle of yield surpass the theoretical one by more than a factor of two. Moreover, we observed a nonlinear dependence of the yield stress versus volume fraction for already moderate magnetic fields. Whereas a multipolar ansatz could diminish the first mismatch, it would not solve the second discrepancy. It might be attributed to the utilized plate-plate geometry of the rheometer, which guarantees a homogeneous field distribution in the sample, but does not apply a homogeneous shear rate. Further measurements will test if a cone-plate geometry diminishes this discrepancy. Finally, the deviations may be attributed to the unclear definition of any yield stress [5]. Here, oscillatory measurements aiming at the viscoelasticity of the network of chains can come to the rescue [6].

Acknowledgments. We thank C. Gollwitzer and G. Jena for measuring fluid parameters and the Deutsche Forschungsgemeinschaft for the financial support via SFB 481, project B9.

\section{REFERENCES}

[1] J. Popplewell, R.E. Rosensweig, J.K. Siller. J. Magn. Magn. Mater., vol. 149 (1995), p. 53.

[2] A.T. SkJeltorp. Phys. Rev. Lett., vol. 51 (1983), p. 2306.

[3] G. Bossis, E. Lemaire. J. Rheol., vol. 35 (1991), pp. 1345-1354.

[4] D.J. Klingenberg, C.F. Zukoski. Langmuir, vol. 6 (1990), p. 15.

[5] J. Schurz. J. Rheol., vol. 36 (1992), pp. 1319-1321.

[6] R. Saldivar Guerrero et al. J. Chem. Phys., (2005), submitted. 